\title{
Aprendizaje Basado en Retos en Lingüística, asignatura de primero del Grado de Filología Hispánica
}

\section{Challenge Based Learning in Linguistics, a first-year course of the Degree in Hispanic Philology}

JuAn PABlo MORA GutiÉRREZ

ORCID https://orcid.org/0000-0002-8614-5117

Universidad de Sevilla

Área de Lingüística General

Departamento de Lengua Española,

Lingüística y Teoría de la Literatura

jmora@us.es

Fecha de recepción: 12-7-2019

Fecha de aceptación: 15-7-2019

DOI: http://dx.doi.org/10.12795/9788447221912.061

Pp.: 1402-1428 
Resumen

Este artículo trata sobre un Ciclo de Mejora en el Aula (CIMA) durante el curso 2018-2019 en la asignatura de primero Lingüística. El CIMA consistió en estructurar la docencia en torno a retos diseñados por el alumnado en equipos que usaron Aprendizaje Basado en Retos con metodología Design Thinking/ECO (ABR con DT/ECO). Al crear y poner en práctica una solución creativa para sus retos, el alumnado tenía la oportunidad de adquirir los conceptos estructuradores de la materia. El conocimiento que se tiene que adquirir en la asignatura cobra así sentido, pues sirve para resolver una necesidad real. Además, tiene que ser compartido. La evaluación se hizo por portafolios. Aunque no se consiguió del todo que se aplicara satisfactoriamente la metodología ECO ni que se cubriera toda la materia del programa de la asignatura, estructurar la asignatura en torno al modelo ABR fue un acierto ya que aumentó la motivación y satisfacción del alumnado.

Palabras clave: Lingüística, Grado de Filología Hispánica, docencia universitaria, experimentación docente universitaria, Aprendizaje Basado en Retos.

\section{Abstract}

This article deals with a Cycle of Improvement in the Classroom (CIMA) in a first-year Introduction to Linguistics course of the Degree in Hispanic Philology during the 2018-2019 academic year. The CIMA revolved around Challenge Based Learning with Design Thinking / ECO (ABR with DT / ECO). The students had the responsibility of acquiring the basic concepts of the subject by using them to solve a challenge that they had to detect and design. This way, the content and knowledge that has to be acquired makes sense because it is created in order to solve real issues. Besides, it has to be shared. The evaluation was done through portfolios. Although the students did not apply ECO appropriately in all the projects and part of the content of the syllabus could not be covered because of time constraints, structuring the course around Challenge Based Learning was a success, because the satisfaction and motivation of the students increased.

Keywords: Linguistics, Hispanic Philology, Higher Education, University Teaching Experimentation, Challenge Based Learning. 


\section{Introducción}

En los dos últimos cursos he participado en dos proyectos de innovación docente que se basan en el modelo de Aprendizaje Basado en Retos (ABR) o Challenge Based Learning con metodología de Pensamiento de Diseño (Design Thinking o DT), que hemos adaptado a ECO (Explorar, Crear y Ofrecer) (Mora, 2017; Herrero-Vázquez y Mora, 2018; Torres-Gordillo, García-Jiménez, Mora-Gutiérrez, Camacho-Taboada y Herrero-Vázquez, 2019). En el curso 20172018, en el proyecto Aprendizaje como proceso de diseño: Innovación universitaria desde el Design Thinking financiado por el III Plan Propio de Docencia de la Universidad de Sevilla participamos once docentes de la US de diversas ramas de conocimiento y más de 600 estudiantes.

Con el asesoramiento de mis compañeros de proyecto apliqué el modelo ABR con DT, en dos asignaturas, Lingüística Aplicada de tercero y Temas de Lingüística de cuarto, ambas del Grado de Filología Hispánica. Una alumna del Máster MASELE lo usó en su TFM bajo mi supervisión. Al final del curso, DT se había transformado en ECO, una adaptación a nuestro contexto. No obstante, no me atreví a usar esta metodología en la asignatura de primero Lingüística. En primer lugar, porque dos de los créditos de la asignatura los compartía con un colega que prefería dar la clase de una forma más tradicional y, en segundo lugar, porque tenía ya asentado un modelo docente basado en la clase invertida o Flipped Learning, con apoyo en las nuevas herramientas tecnológicas, Aprendizaje Basado en Proyectos y Aprendizaje Cooperativo (Mora, 2014, 2015, 2017). Tanto el alumnado de primero como yo estábamos alcanzando altos grados de satisfacción con este modelo que había implementado por primera vez en la asignatura completa en el curso 2013-2014. Desde entonces he venido perfeccionando esta nueva manera de llevar a cabo la docencia con el apoyo de las redes docentes en las que me he ido integrando, entre ellas la REFID de la US. 
Como evidencia del aumento de la satisfacción del alumnado baste señalar que en la pregunta 18 "En general, estoy satisfecho/a con la actuación docente desarrollada por este/a profesor/a" de la encuesta oficial de la US en el curso 2011-2012, en el que no había iniciado todavía el camino hacia la innovación docente, obtuve un 2,58 sobre 5 (n=38). En 2012-2013, en el que implementé por primera vez la evaluación por medio de portafolios e inicié la transformación hacia una docencia centrada en el aprendizaje, probando el modelo Flipped Learning durante las últimas semanas del cuatrimestre, obtuve 3,71 ( $n=17)$. En 2013-2014 en el que por primera vez impartí toda la asignatura con ese modelo de clases al revés bajé a un 3,05 $(n=37)$, mientras que en el 2014-2015 subí al 3,78 ( $n=63)$ y en el curso 2017-2018 obtuve 4,17 ( $n=36)$. La subida en la satisfacción del alumnado es aun más notable si se comparan estos resultados de Lingüistica de Filología Hispánica con lo obtenido en esta misma pregunta en los grupos de primero del Grado de Estudios Ingleses.

En la figura 1 se puede ver la comparación y se puede observar mejor el avance en la satisfacción del alumnado. En el curso 2013-2014 hubo un retroceso importante, pues cometí el error de evaluar no solo a través del portafolios, sino también con un examen obligatorio que valía el 50\% de la nota. El alumnado no perdonó la incongruencia, que suponía haber cambiado la docencia radicalmente manteniendo como herramienta de calificación el tradicional examen final obligatorio. 


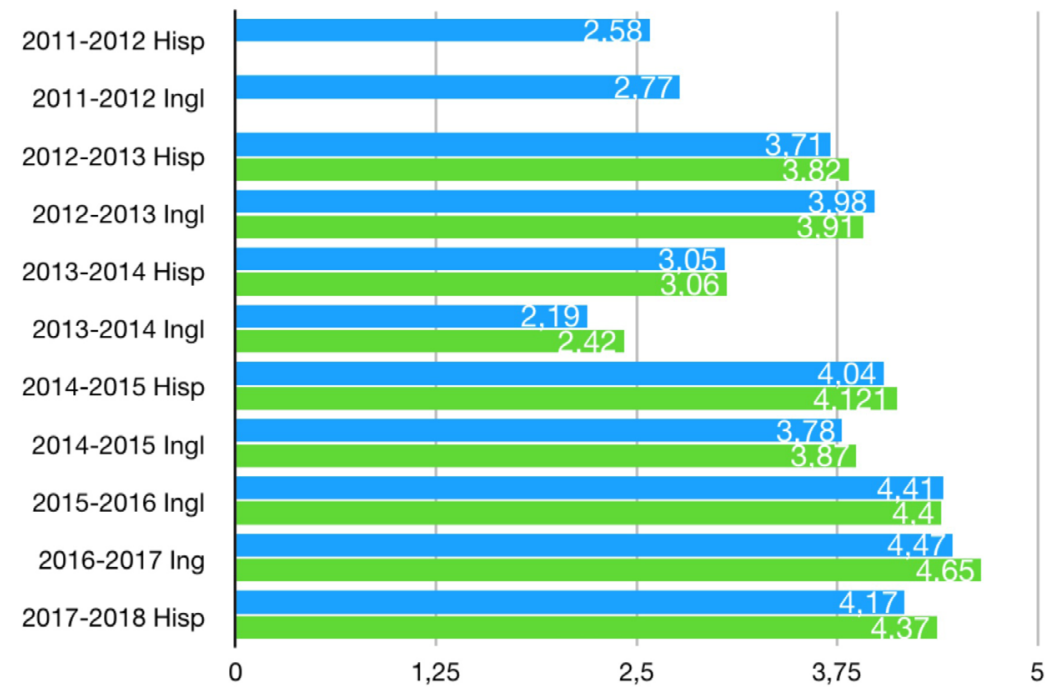

Figura 1. Evolución de los resultados de satisfacción del alumnado. En azul se recoge la pregunta 18 sobre la satisfacción con la docencia y en verde la media ponderada global de todas las preguntas de la encuesta. Se puede acceder a las encuestas y a más información en la entrada de blog que sirve de repositorio para este artículo: https://ipmoragu.wordpress.com/ introl2019/. Fuente: elaboración propia

En el curso 2017-2018, el alumnado de Lingüística recibió formación sobre lo que era ABR con DT/ECO, por si algún equipo quería usarlo en el proyecto que llevaban a cabo de forma cooperativa fuera del aula. Solo un equipo optó por usarlo, pero sin éxito, por haberse decidido a hacerlo a última hora, cuando ya habían terminado las clases.

Finalmente, en el curso académico 2018-2019 me he decidido a usar este modelo docente en primero, en el marco del proyecto de innovación docente ECO en la Educación Superior: docencia inspirada en el entorno, continuación del anterior. Ha sido sobre todo por el ejemplo que me proporcionaron dos de los compañeros de proyecto que 
sí que se habían atrevido a ponerlo en práctica en materias de primero en 2017-2018. También me ha impulsado la constatación de que a pesar de todos los esfuerzos resulta muy dificil que el alumnado de primer curso adquiera de forma profunda y duradera algunos de los conceptos estructuradores, por resultarles demasiado dificiles, abstractos y ajenos a sus intereses y a su vida. Con todo, lo que más me ha motivado a cambiar de enfoque ha sido la comprobación estos últimos años de que cuanto más control, responsabilidad, autonomía y confianza deposito en el alumnado y más oportunidades doy para construir el conocimiento de forma autónoma, los resultados son mejores puesto que la satisfacción y motivación aumentan.

\section{Diseño previo del CIMA}

El objetivo de la asignatura de 6 créditos Lingüística, obligatoria en los Grados de Filología, es ofrecer una introducción de todos los niveles y ámbitos del estudio del lenguaje y las lenguas abordados desde las ciencias del lenguaje. Hay un total de diez grupos de los cuales tres corresponden al Grado de Filología Hispánica. El grupo de este CIMA ha sido el de tarde. Cuando comenzó el cuatrimestre habría en torno a 30 estudiantes en lista, que se incrementaron a 46 tras la matriculación de 16 estudiantes foráneos.

Antes incluso de comenzar las clases, informé al alumnado de que tendría la última palabra en cuanto a la metodología que usaríamos. Le mandé información a través de la plataforma de enseñanza virtual sobre los dos modelos docentes en liza: Flipped Learning y ABR con DT/ECO. Si optábamos por ABR, la inmensa mayoría de las sesiones de clase se dedicarían al trabajo en equipo para resolver los retos que tendrían que diseñar y desarrollar. Les sugerí que podríamos hacer una prueba de uso de ABR con DT/ ECO durante las tres primeras semanas del cuatrimestre 
con un primer mini reto centrado en la diversidad lingüística y cultural en el marco del Año Internacional de las Lenguas Indigenas de la UNESCO https://es.ivil2019.org. El objetivo sería aprender sobre la metodología DT/ECO llevando a cabo en equipo un reto que, además, serviría para construir conocimiento de algunas de las cuestiones centrales en el estudio del lenguaje y las lenguas. El conocimiento creado tendría que ser ofrecido a la sociedad durante la Fiesta de la Historia. (https: / / fiestadelahistoria. wordpress.com). Los equipos se conformaron según intereses comunes con la única restricción de que tenía que haber alumnado español y extranjero.

Las actividades que creó el alumnado para la Fiesta de la Historia fueron un éxito, como se puede observar en este vídeo resumen, publicado en el canal de YouTube de la Facultad de Filología: https://youtu.be/VG9WjDfSHDw. Mi alumnado de primero participó en este evento junto al alumnado de Temas de Lingüística de cuarto y al alumnado de la asignatura del Máster MELLC, Procesos de Globalización Lingüística. También participó alumnado de Lingüística de primero del Grado en Lengua y Literatura Alemanas y del Grado en Estudios Ingleses (Camacho, 2019).

Para que pudieran decidir mejor si querían que siguiéramos usando ABR con DT/ECO el resto del cuatrimestre, invité al alumnado de primero al \#ECOshowUS del 16 de enero de 2019, que se celebró en el aula Magna de nuestra facultad y en el que podían ver lo que había conseguido con sus proyectos el alumnado de tercero de la asignatura de primer cuatrimestre Lingüística Aplicada y el de las otras ramas de conocimiento que también participaban en el proyecto de innovación docente. Se puede ver un vídeo resumen de este evento: https://youtu.be/ak1MY4p93iM. También les pasé el vínculo del padlet de la asignatura Lingüística Aplicada: https://padlet.com/imora4/ax4pbgolrwfo. Añadí al padlet otros proyectos que se habían desarrollado con ABR en cursos anteriores y que podrían 
servir de modelo, como los proyectos de documentación y revitalización lingüistica de la lengua haida y de la lengua mixteca (proyecto de Investigación-Acción ndatiaku tu'un savi iniciado con el alumnado de primero en 2014).

Daba la casualidad de que la galardonada película Roma de Alfonso Cuarón tenía como protagonista a una mujer que hablaba la lengua mixteca (el tu'un savi o lengua de la lluvia). Es por ello que pedí permiso a Alfonso Cuarón y a su productora para llevar a cabo un Romatón en la US, que nos permitiera reflexionar sobre cómo la película plantea diversos temas relacionados con la lingüística, como la diversidad lingüística y cultural de México, la subordinación de unas lenguas con respecto a otras y la violencia verbal. Tras la participación en la Fiesta de la Historia, se votó en clase y todo el alumnado optó por el modelo ABR con DT/ ECO, excepto una alumna que ya lo había experimentado satisfactoriamente el cuatrimestre anterior en Lingüística Aplicada, razón por la que quería probar un modelo distinto.

El modelo de ABR con metodología DT/ECO supone un cambio disruptivo sobre cómo se lleva a cabo la docencia universitaria. Su principal característica es que la docencia no se centra ni en el docente ni en los contenidos, como en la docencia tradicional, ni siquiera en el alumnado, como en muchas metodologías innovadoras, sino en la sociedad y en sus necesidades. El alumnado, organizado en equipos, tiene que detectar una necesidad de la sociedad para convertirla en un reto a través del cual el conocimiento y contenido de la materia que va a adquirir y aplicar durante el cuatrimestre le tiene que servir para crear una solución innovadora y original. El foco de la docencia sale del aula para situarse en la sociedad y en los desafios a los que esta se enfrenta. Esta metodología tiene puntos comunes con el Aprendizaje Servicio, con la diferencia de que en la mayoría de las ocasiones en ApS el reto está fijado de antemano mientras que en el ABR es el alumnado el que descubre y explora una necesidad de la 
sociedad para diseñar un reto ad hoc para ofrecer soluciones creativas a las personas a las que va a servir y de las que va a aprender durante toda la experiencia.

Con el acuerdo del alumnado con respecto al cambio de modelo docente pude finalmente diseñar el CIMA. Se decidió que el alumnado definiría un reto global y un colectivo al que dedicaría sus esfuerzos, pero a diferencia de lo que se hacía en las asignaturas de tercero y cuarto, en las que el alumnado se centraba y trabajaba en un único reto todo el cuatrimestre, en la asignatura de primero se llevarían a cabo tres mini retos, uno para cada uno de los niveles de descripción lingüística. Así habría un primer reto que se dedicaría al ámbito de la Fonética y Fonología, las ramas de la lingüística que se ocupan de los fonemas y de los sonidos del habla, un segundo reto se centraría en el ámbito de la Morfología y la Sintaxis, la parte más formal de la lingüística, que se ocupa de las palabras y las oraciones, y un tercer reto dedicado a la Semántica y la Pragmática, las dos ramas que se ocupan del significado y de los textos y enunciados. De esta forma se tratarían todas las disciplinas fundamentales de la lingüística y se cubrirían los conceptos básicos de la materia. Se decidió que las sesiones de clase se dedicarían al trabajo en equipos en torno a los retos. Habría clases también para las presentaciones de los avances de los equipos, clases para debates, así como clases a las que vendrían invitados que presentarían temas de interés o que asesorarían a los equipos.

Para garantizar que el alumnado profundizara en los retos de los demás y para que se fueran cubriendo todos los ámbitos de la asignatura, se estableció que cada semana habría que, o bien escribir un ensayo de opinión o bien llevar a cabo una serie de ejercicios prácticos obligatorios que presentaran desafios al alumnado y que obligaran a tener que investigar y aplicar algunos de los conceptos claves y estructuradores de los diferentes niveles de la materia. También se mantuvo de años anteriores 
la opción de llevar a cabo a través de las redes sociales la recogida de ejemplos de neologismos, lapsus linguae, de usos discriminatorios y prejuiciosos del lenguaje, de errores que cometen los aprendices de una segunda lengua y de noticias que aparecieran durante el cuatrimestre sobre cualquier tema relacionado con la Lingüística. Además, el alumnado se responsabilizaría de explorar y consolidar su conocimiento de la materia a través de los vídeos de clases magistrales grabados por el propio docente en inglés y español o por medio de los manuales recomendados o a través de la bibliografia y las fuentes de información específicas descubiertas por los equipos para hacer frente a sus retos. Por último, se acordó que la evaluación se llevaría a cabo por medio de autoevaluación, de evaluación por pares, a través de cuestionarios y de un portafolios final que recogería las evidencias de aprendizaje seleccionadas, la reflexión sobre ellas, un diario reflexivo de clase y la valoración de todo lo acontecido en la asignatura. Se enfatizó que la asignatura, al ser de seis créditos, requería un trabajo de unas 150 horas como máximo, de las que se recomendaba emplear en torno a 50 para el reto o proyecto en equipo, otras 50 para los ensayos, tareas obligatorias y preparación de clases y debates, y las restantes 50 a las lecturas, visionado de vídeos, asistencia a eventos y actividades de interés, presenciales o en línea, y a la elaboración del portafolios final.

\section{Aplicación del CIMA}

En el curso 2017-2018, el grupo de tarde de primero tuvo 66 matriculados, de los que 21 no participaron de la evaluación continua ni se presentaron al examen. El curso 2018-2019 únicamente se matricularon 46 y solo ocho han obtenido un no presentado. De cuatro de ellos no he sabido nada ni los he conocido, los otros cuatro no han podido seguir la asignatura de forma constante ni presentarse al examen por diversos motivos. Cabe destacar 
que uno de ellos es un alumno que estaba terminando el Grado de Derecho, además de estar cursando primero de Filología. Este alumno vino a casi todas las clases y participó activamente, proponiendo y moderando el debate sobre las lenguas de España, pero consideró que no disponía del tiempo necesario para el trabajo en equipo y todo lo que requería la asignatura. En un principio había optado por la evaluación a través del examen final, pero le entusiasmó tanto lo vivido en clase que decidió no presentarse al examen para matricularse de nuevo en Lingüistica el curso 2019-2020 y poder así participar plenamente en la experiencia en la que había visto desarrollarse y crecer tanto a sus compañeros. Solo dos estudiantes optaron por la evaluación a través del examen final, por encontrarse trabajando. Los 36 restantes son los que han participado en la evaluación continua y en este CIMA. De ellos, 16 son extranjeros con una gran diversidad de origenes (tres chinos, tres rusos, cinco italianos, una francesa, una austríaca y tres alemanes).

Para llevar a cabo sus retos se agruparon en siete equipos, algunos en continuidad con los que se habían creado para el mini reto de la Fiesta de la Historia. Tres de ellos optaron por un reto basado en la enseñanza de español como lengua extranjera (ELE) a hablantes de otras lenguas:

- ELE para ruso hablantes, con un equipo de cinco personas formado por los tres alumnos rusos y dos españolas.

- Español para sinohablantes, con un equipo formado por los tres alumnos chinos y una alumna de habla materna española. Se denominaron proyecto SinoELE.

- Español para hablantes de alemán, con cuatro alumnos españoles y tres alemanes, incluida una de ascendencia turco-kurda.

El cuarto equipo se dedicó a la comparación de varias lenguas con el objetivo también de enseñar y traducir mejor entre lenguas. Se centraron en español, francés, 
italiano, alemán e inglés, pero también trataron otras lenguas. Este equipo estaba integrado por dos españolas y tres Erasmus, uno de Italia, otra de Austria y la tercera de Francia, aunque de ascendencia española. En el primer reto se llamaron "Pronúnciate", en el segundo "Exprésate".

Los otros tres equipos eligieron temas singulares. Un equipo formado por tres italianos y dos españolas eligió el tema Teatro y Lingüística. Otro equipo formado por una Erasmus italiana de ascendencia magrebí y cuatro españolas se centró en las personas con TEA (Trastorno del Espectro Autista) y adoptaron el nombre Inside Out. Por último, un equipo formado por cinco alumnas españolas se centró en investigar el tema de la conexión entre la dislexia y la lingüística. Decidieron llamarse a sí mismas Las Persianas, por la broma extendida entre los disléxicos en la que dicen que "los dixélicos también somos persianas", cambiando "disléxico" por "dixélico", y "personas" por "persianas".

Aunque no dio tiempo para llevar a cabo el tercer reto previsto sobre Semántica y Pragmática, los logros y el avance de todos los equipos y del alumnado individualmente han sido muy notables. Uno de los motivos puede haber sido que se ha depositado en ellos mucha confianza y altas expectativas proporcionándose un entorno seguro en el que podian equivocarse y aprender de los errores. También ha habido bastante flexibilidad para conseguir una buena adaptación al ritmo de trabajo de cada equipo, a la complejidad de los diversos retos y a las necesidades y circunstancias de las personas y los equipos que los afrontaban. Además, se ha creado un buen ambiente de trabajo tanto en clase como fuera de clase. Ha contribuido también que hayan tenido que llevar a cabo varias presentaciones en clase y que se les haya dado la oportunidad de compartir lo que creaban en eventos fuera del aula. La primera presentación en clase fue tras haber ofrecido sus primeros retos a la sociedad en la Fiesta de la 
Historia. Otra segunda tras el reto sobre Fonética y Fonología, y una tercera tras la fase de Morfología y Sintaxis. Por último, hubo una presentación final para elegir los mejores proyectos que representarían a la clase en el \#ECOshowUS del proyecto de innovación docente en la sede del CICUS el 5 de junio.

Además, cada persona ha tenido que escribir, al menos, 6 breves ensayos de opinión. Tenían que elegirlos entre más de 30 posibles temas que estaban relacionados con los debates llevados a cabo en clase o con los proyectos. Algunos habían sido propuestos por el profesor, pero la mayoría provenían de las propuestas del propio alumnado o de las preguntas y cuestiones que surgían en el desarrollo de los retos. Para estos ensayos no había fecha específica, sino que tenían todo el cuatrimestre para subirlos a la plataforma, según sus intereses y sus obligaciones. Si que había fecha de entrega de los ejercicios prácticos obligatorios, que fueron los siguientes:

1. Su biografía "lingüística", que constituiría la primera sección de su portafolios individual final.

2. Una tarea de transcripción fonética de una lengua desconocida para ellos. Se les pidió que transcribieran lo que pudieran durante 45 minutos de la escena de la Casa del Pavo de la película Roma, en la que Cleo, la protagonista, habla con su compañera Adela en mixteco, pero intercalando también frases en español. Tras intentar la transcripción se tenía que escribir una reflexión sobre por qué era relativamente fácil transcribir las frases en español, pero muy complicado el diálogo en mixteco, a menos que se tuviera el oído entrenado para la labor de transcripción fonética.

3. Una tarea de reforma de la ortografía de la lengua española que llevo usando satisfactoriamente desde hace más de seis cursos. 
4. Una tarea de evaluación por pares de las primeras presentaciones en clase de los siete equipos tras el primer reto del nivel fonético-fonológico. Además de evaluar la presentación de su propio equipo y las de los demás, se les pidió que evaluaran críticamente una presentación que su docente había llevado a cabo en el EABE 2019 de Salobreña, precisamente sobre el uso de ABR con DT/ECO en Lingüística. Esta presentación no había salido todo lo bien que el docente esperaba, por haberla llevado a cabo de manera frontal con poca participación e implicación del público asistente, docentes innovadores de todos los niveles educativos. El objetivo de esta tarea era hacer ver al alumnado que de lo que más se aprende es de lo que sale mal, si se sabe identificar y aprovechar.

5. Una tarea sobre sintaxis y su interfaz con la semántica que permite reflexionar y aprender sobre algunos de los conceptos claves de este nivel de la lingüística.

6. Una tarea relacionada con el visionado en hora de clase de la película Roma en el Romatón que se organizó en la US. La tarea consistía en escribir un ensayo sobre algunas de las cuestiones que se habían tratado en las mesas de debate.

7. Un taller conceptual sobre gramática contrastiva.

8. Una tarea sobre el nivel semántico pragmático.

Además, se llevaron a cabo cuatro debates a propuesta del alumnado. Estos debates fueron grabados en vídeo, para facilitar al alumnado Erasmus o al que hubiera faltado, poder verlos de nuevo. Los debates trataron sobre los siguientes temas:

1. Debate sobre las lenguas de España y su convivencia. Por su perspectiva política y jurídica, lo moderó el alumno que estaba terminando Derecho junto a otros dos compañeros. 
2. Debate sobre el sexismo lingüístico y el lenguaje inclusivo. Lo moderó el equipo de la clase de Temas de Lingüística de cuarto que estaba trabajando sobre violencia verbal. En ese equipo había dos alumnas Erasmus que estaban también cursando la asignatura de primero.

3. Debate sobre la dislexia, ideado y moderado por el equipo Las Persianas, que tenía como tema la dislexia en contextos multimodales y multilingües.

4. Debate entre profesores organizado por el área de lingüística general que trató el tema de la $\mathrm{Hi}-$ pótesis Sapir-Whorf o de relativismo lingüístico. El alumnado propuso las preguntas y moderó el debate.

Se puede acceder a las grabaciones de los debates, al enunciado de las tareas, a algunos de los ensayos y en general a todos los vínculos que aparecen en este texto y a información complementaria en la entrada de blog que sirve como repositorio de este artículo: https://ipmoragu. wordpress.com/introl2019.

También se invitó a clase a diversos expertos y a otros docentes para dar feedback al alumnado sobre su trabajo y algunas nociones básicas de temas relevantes. Vinieron representantes de la asociación Dislexia-Sevilla, para asesorar al equipo Las Persianas, un actor de teatro para ayudar al equipo de Teatro y Lingüística, el creador y coordinador del proyecto ndatiaku tu'un savi para hablar sobre diversidad lingüística (https://youtu.be/H9Lyh7YoDol) y para llevar a cabo actividades de doblaje y subtitulación basadas en la película Roma y en el fragmento que se había transcrito fonéticamente (actividades que llevaremos a la próxima Noche Europea de los Investigadores), una profesora jubilada experta en el tema del componente afectivo en la enseñanza de lenguas y algunos compañeros del proyecto de innovación. También pasaron por la clase antiguos alumnos que habían trabajado con ABR para contar 
brevemente sus proyectos y para reflexionar sobre los problemas con los que se habían encontrado durante el trabajo en equipo.

El 2 de mayo fuimos como clase a la sede del CICUS para participar en el Romatón (https://cicus.us.es/romaton). Aunque parte del alumnado se había mostrado contrario a ver la película Roma, por la fama que tenía de lenta y aburrida, lo cierto es que tras verla en pantalla grande pudieron apreciar la gran obra de arte que es. El jueves 23 de mayo, tres de los equipos, junto con cinco de la asignatura de cuarto, acudieron a la MUAC, la Muestra Anual de Comunicación. El equipo Las Persianas tuvo mucho éxito con la actividad que llevaba sobre dislexia. Un docente de Educación las invitó a sus clases y además fueron entrevistadas en el programa En Primera Persona de RNE. Intervienen al final del programa emitido el 10 de junio.

La primera semana de junio (última de clase), tuvieron lugar las presentaciones finales de los proyectos. Se pidió a los equipos que las llevaran a cabo en menos de cinco minutos y en formato showroom para que se pudiera determinar qué equipos se ganaban el privilegio de representar a la clase en el \#ECOshowUS final del proyecto de innovación docente. En este enlace pueden verse las presentaciones de los siete retos:

\section{h t t p s: / / w w w. y o u t u b e.com/ playlist?list=PL7nyPp6kDT5nwTbsf-4yuWanG1GzRwVLI}

Si se comparan con las primeras que los equipos llevaron a cabo, se puede apreciar todo lo que el alumnado ha crecido en la clase, no solo en cuanto a los contenidos adquiridos de la materia, sino sobre todo con respecto a las competencias transversales de capacidad para comunicarse, trabajar en equipo, enfrentarse a las dificultades y aprender de los errores. 
El equipo cuya presentación quedó en primer lugar fue el equipo SinoELE. Es justo el equipo al que peor le había salido la presentación anterior en clase sobre el reto del nivel morfosintáctico. Se puede comprobar que supieron aprender del error. En segundo lugar quedó el equipo Las Persianas y en tercer lugar el equipo Teatro y Lingüística. Los tres fueron excelentes representantes de la clase en el \#ECOshowUS. En los vídeos resumen aparecen como protagonistas el docente de la asignatura y dos de las alumnas de la clase. En el siguiente enlace se puede ver el realizado por el gabinete de comunicación de la US https://youtu. be/Ol2xJA 90kM y en este otro el resumen creado por la productora Mirada Global https://youtu.be/mk1vk35/8HE.

Vale la pena ver lo acontecido en el \#ECOshowUS, pues pone de manifiesto lo efectiva que es la metodología ECO. Muchas de las presentaciones están en este enlace:

h t t p s: / / w w w. youtube.com / playlist?list=PL l-eodz6U7eLzxqPQghMQzstcnWINL39

A la última clase asistieron solo doce personas por ser viernes. Se aprovechó para llevar a cabo un grupo de discusión de evaluación de lo que había sido la asignatura. Se dividió al alumnado en dos equipos de seis personas. Cada equipo creó un prototipo visual de lo que había representado la asignatura y de lo que habían aprendido. Con ese apoyo de pensamiento visual se dedicó la clase a la evaluación de lo que había sido la clase y lo que pensaban sobre el modelo docente utilizado: https://youtu. be/-eYskaHblic

En la grabación se puede ver el buen ambiente de la clase y la satisfacción del alumnado con todo lo que había acontecido y todo lo que sentían que habían aprendido. Las únicas quejas se refirieron al excesivo número de horas de trabajo que decían que habían tenido que dedicar a la asignatura y a la falta de contenido teórico en clase al inicio de cada mini reto. 
La fecha limite de entrega de los portafolios individuales era el 10 de junio de 2019, el mismo día del examen. En el repositorio se pueden consultar los portafolios para los que se tiene permiso. Si se lee cualquiera de ellos, se podrá percibir el alto grado de satisfacción del alumnado y el alto grado de implicación y esfuerzo dedicado a la asignatura. A partir de esa fecha, se llevaron a cabo en las horas de tutoría entrevistas de evaluación del trabajo en equipo entre el docente y todos los miembros de los equipos. En ellas se puso de manifiesto el alto grado de satisfacción con el trabajo en equipo y se discutieron los problemas que habían surgido y cómo se habían resuelto. Este alto grado de satisfacción es similar al alcanzado el curso pasado en todas las áreas de conocimiento del proyecto de innovación (Torres-Gordillo et al., 2019).

Se ha dado un nuevo plazo hasta el 15 de julio de 2019 para que el alumnado pueda presentar versiones mejoradas de sus portafolios y para que pueda evaluar portafolios de otro alumnado de la clase y también los portafolios grupales de la asignatura de cuarto. A su vez, al alumnado de cuarto se le ha pedido que valore portafolios de primero y dé consejos que puedan servir durante el resto de la carrera al alumnado de Lingüística.

\section{Evaluación del CIMA}

Hay dos cuestiones que no han funcionado en la puesta en práctica del CIMA. En primer lugar, por falta de tiempo no dio lugar a realizar el tercer reto sobre el nivel de Semántica y Pragmática. Así pues, estas dos ramas de la lingüística solo pudieron tratarse en uno de los ejercicios prácticos obligatorios. La segunda está relacionada con la propia metodología ECO. Apenas se enfatizó la parte creativa, pues no hubo ocasión ni de crear prototipos ni de llevar a cabo lluvia de ideas. Tampoco hubo conciencia en algunos equipos de que se estuviera trabajando para 
otras personas. Falló, pues, la fase Empatiza, pues no se insistió lo suficiente sobre ella. Estas son las debilidades principales que se han de mejorar el curso que viene. Otra cuestión que debería mejorar es cómo atender a alumnado que no use Internet regularmente o no lo tenga en casa. En este CIMA he tenido una alumna que apenas usa Internet por no tenerlo en casa y no he sabido proporcionarle buenas alternativas para el acceso a los materiales y actividades centrales de la materia.

No obstante, lo positivo predomina. Para todos sin excepción la experiencia ha sido enormemente enriquecedora. Hemos aprendido mucho sobre la dislexia, el alumnado con TEA, el teatro y su relación con la lingüística, sobre chino, ruso y las demás lenguas en las que se han explorado en clase y de todas las actividades y soluciones creadas por el alumnado. La mejor forma de mostrar el enriquecimiento mutuo es cediéndole la palabra al alumnado que lo ha protagonizado. Conviene advertir que se dejarán los errores del español escrito por el alumnado extranjero, ya que son reflejos de su interlengua.

En primer lugar, se recomienda ver este vídeo en el que las cinco Persianas cuentan su experiencia y todo lo que han trabajado y aprendido en su viaje al mundo de la dislexia: https://youtu.be/IsGolKwoBBM En segundo lugar quiero destacar estas palabras que cierran la valoración que me mandó el domingo 14 de julio la alumna que no usa apenas Internet por no tenerlo en casa. Esta alumna había sido la más crítica en su portafolios final y sentía que en esta ocasión tenía que destacar lo positivo:

Gracias a usted, he conocido a gente muy diferente, he visto que cada persona es un mundo, por fin, he aprendido a trabajar en grupo y aprender que cada error es un paso. Por todo esto y por muchísimas cosas más, GRACIAS, ha sido un placer dar clases con usted y espero verle en cuarto.

Jornadas de Formación e Innovación Docente del Profesorado | № 2 (2019) Esta obra se distribuye con la licencia Creative Commons 
En el documento que me envió lleva a cabo una evaluación muy crítica de tres de los portafolios de sus compañeras de clase y de tres de los portafolios grupales de cuarto. Cierra el documento con una sección que titula Aclaración y en la que dice que en su portafolios del 10 de junio se había centrado en las críticas porque sabía que el resto de la clase destacaría lo positiva y satisfactoria que había sido la experiencia.

Las siguientes citas que se han tomado de los portafolios finales muestran eso precisamente. En ningún otro curso las opiniones del alumnado han sido tan unánimemente favorables, ni ha habido tan pocas críticas. Una alumna española que ha cursado este cuatrimestre tanto la asignatura de primero como la de cuarto escribe lo que sigue en el portafolios individual opcional para la asignatura de cuarto, enviado la madrugada del 11 de julio de 2019:

He tenido la suerte de trabajar con este método por partida doble. $Y$, sinceramente, ha resultado ser el mejor cuatrimestre de todos los que llevo cursados en la carrera. Eso sí, no he parado de trabajar ni un solo segundo, pero mirando hacia atrás, me doy cuenta de todo lo que he llegado a aprender en tan poco tiempo.

Esta alumna formó parte del equipo Las Persianas y se muestra satisfecha de que tras llegarle una alumna con dislexia a la academia en la que trabaja pudo atender sus necesidades gracias a lo aprendido en el proyecto:

Por tanto, he de decir que, sin duda alguna, los proyectos que he realizado tanto en primero como en cuarto con esta metodología me han ayudado en mi día a día con creces. Estoy consiguiendo que a mis alumnos de la academia (tanto de secundaria como bachillerato y pruebas de acceso) les guste, entiendan y aprendan la asignatura de lengua y literatura a través de las actividades expuestas, explicaciones dinámicas e interactuaciones entre ellos. También, tuve la suerte de poner en práctica lo aprendido en el proyecto de primero, y tras las notas de fin de curso, puedo decir que sé tratar con alumnos con dislexia y ayudarlos a aprender haciéndoles olvidar sus dificultades.

Jornadas de Formación e Innovación Docente del Profesorado | № 2 (2019) Esta obra se distribuye con la licencia Creative Commons 
Señala, además, que esta metodología ha conseguido despertar su creatividad:

Además del aprendizaje académico que he expuesto, he aprendido a nivel personal, que la creatividad que creía perdida nunca me abandonó. También, que puedo llegar a aportar muchísimo a la comunidad que nos rodea, que con paciencia y constancia se consiguen muchos beneficios. A creer y confiar más en mí misma. Y, por último, que el trabajo y la ilusión siempre traen consigo una recompensa.

Y termina resaltando que la clave ha estado en la confianza depositada por el docente:

Por todo ello, no me cansaré de dar las gracias al profesor por valorar el esfuerzo y el trabajo que realizan sus alumnos, por transmitir su pasión por la lingüística, por su dedicación y comprensión, y, sobre todo, por confiar siempre en cada uno de nosotros.

Una alumna de origen chino del proyecto SinoELE destaca en su portafolios cómo se convirtieron en un auténtico equipo después de superar todos los retos y solventar las dificultades:

Me he dado cuenta de que lingüística está tan cerca de mí y puede aplicar en muchos terrenos. Me he costado mucho tiempo terminando todos los ejercicios obligatorios y los ensayos, sobre todo nuestra presentación en equipo. Como hemos aprendido mucho de la primera presentación, nos dedicamos más tiempo fuera de clase juntos preparándola para que saliera mejor. Pues después de varias presentaciones, he comprendido cómo sentirme menos nerviosa en la presentación y presentar todo de manera mejor.

Como teníamos que leer varias tesis, he practicado mucho la habilidad de sacar informaciones. Tuve muchos trabajos en equipo los dos años pasados, pero nunca he tenido la experiencia de que todo el grupo dedicó un proyecto todo el cuatrimestre. Así que después de todos retos que hemos superado juntos, ahora somos de verdad un equipo que apoyamos mutuamente.

Una alumna Erasmus del equipo Exprésate, que formó parte además del equipo sobre violencia verbal en la asignatura de cuarto, escribió lo siguiente sobre ECO y el trabajo en equipo: 
En general, la experiencia ECO ha sido muy enriquecedora para mí, ya que nunca antes he trabajado con esa metodología y, la verdad, al principio dudaba de que realmente sirviese para aprender. Las (pocas) experiencias que tenía en trabajar en grupo siempre resultaban en lo mismo: una persona (o dos) se ocuparon de todo y el resto del equipo se aprovechó de su trabajo. Como la metodología ECO funciona a través de retos y tiene pautas muy claras, me resultó mucho más fácil incorporarme en el grupo y poder avanzar. Aunque al principio me costó tiempo entender todos los pasos de esa metodología, al final me pareció todo muy lógico y entendible, es más, las fases de ECO son comparables con aquellas que hay que recorrer al trabajar, por ejemplo, en un proyecto laboral. Como yo soy una persona a la que cueste mucho adquirir contenido teórico si no encuentro en ello una aplicación concreta o un sentido superior, la metodología me vino genial, ya que teníamos una aplicación concreta para todos los niveles de la lingüística y eso me ayudó mucho a la hora de aprender sobre los diferentes conceptos.

\section{Y esto sobre el docente:}

El profesor ha tenido un papel fundamental en todo ese proceso de aprendizaje, ya que no tan solo logró generar un ambiente (casi) familiar en clase, sino también siempre entró el aula con ánimo y alegría y supo transmitir esa energía a sus alumnados. Fuera de clase, el profesor siempre respondió con paciencia a las preguntas o dudas que surgieron en clase y supo resolver cualquier problema. Sinceramente, a veces, las grabaciones en clase generaron un ambiente un poco incómodo, ya que a muchos alumnos les da mucha vergüenza que los graben y no se atreven decir lo que realmente piensan, ya que sus palabras quedan grabadas y el profesor puede re-escucharlas cuando quiera. Por otra parte, me parece genial la idea de sacar videos de las presentaciones, ya que fomenta mucho la capacidad crítica. Además, creo que una introducción teórica (aunque sea muy breve) del nivel lingüístico que se va a tratar en continuación a través de la metodología ECO, serviría mucho para que los alumnados sepan orientarse mejor en sus proyectos y tendrán tiempo para llevar a cabo todas las fases ECO.

\section{Otra alumna Erasmus del equipo de ELE para alum- nado alemán escribió:}

¿Qué es ECO? Todos conocemos la expresión "hacerse eco de" como la contribución a que un asunto sea aceptado o tenga resonancia, repercusión, difusión. Y si hablamos de lo que es el "eco", podríamos decir que se trata de la "repetición de un sonido producida al ser reflejadas sus ondas por un obstáculo". El eco tiene lugar cuando hay elementos que vibran en la misma onda. Metafóricamente hablando, imaginaos un docente que vibra en la misma onda que sus estudiantes: las ondas en 
una misma frecuencia se suman y se amplifican produciendo eco, mientras que si permanecen en frecuencias distintas se restarán y el efecto será totalmente el contrario, ni siquiera se oirán. Aplicando la metodología ECO, tenemos la oportunidad de hacernos eco de nuestro entorno, de cubrir nuestras propias necesidades, y la de otras personas.

En este parte quiero decir gracias a Juan Pablo, para darnos la oportunidad de sentirnos como futuros profesores. Yo siempre estoy a favor de este método porque vale a mucho más. Es importante que los estudiantes investigan por su mismo y no se dice todo el profesor. En nuestra clase de Lingüística no había un profesor que era Juan Pablo, teníamos casi 30 profesores de diferentes países con diferentes métodos y estudios.

Este es uno de los puntos centrales. Gracias a ABR con DT/ECO, no hubo una única voz ni se escuchó una única historia, sino que cada persona construyó su propio relato y contribuyó al enriquecimiento de todos.

Una alumna española del equipo Inside Out, que trabajó para las personas con trastorno del espectro autista TEA, lo cuenta así:

Como reflexión del curso diría que ha sido una de las pocas asignaturas, por no decir la única en todos los años de instituto y carrera que llevo en la que se ha fomentado tantísimo los trabajos en grupo y la participación de los alumnos en clase, además de que hemos hecho muchísimas actividades, hemos conocido a muchísimas personas, actores, profesionales de la lengua, profesores de otras universidades, antiguos alumnos etc., además de hablar de temas muy variados y hemos aplicado la lingüística a muy diversos temas, sinceramente me ha parecido una experiencia muy enriquecedora y sobre todo diferente, la metodología ECO es una metodología que conlleva mucho trabajo pero que te permite explorar muchos ámbitos distintos tanto de la enseñanza como de la lengua y sobre todo me parece un método innovador y que nos obliga a relacionarnos unos con otros, a conocer otras lenguas y otras culturas, y a tener que investigar, ir a centros, hacer entrevistas etc., ha merecido mucho la pena, y lo mejor de todo es que todas las actividades que hemos hecho no nublan el tema central de la asignatura que es la lingüística sino que aprendemos por nosotros mismos y a través de otros mecanismos que no son los habituales, somos nosotros los que hemos ido dando las clases presentando nuestros proyectos de investigación y hablando de la lingüística en distintos ámbitos de la vida, además creo que gracias a la metodología, ahora estamos todos mucho más sueltos a la hora de hablar en público y de expresarnos delante de una cámara o 
delante de un público amplio, que al fin y al cabo es lo que vamos a tener que hacer en un futuro cuando demos clases y estoy segura de que si me dan la oportunidad aplicaré esta metodología con mis futuros alumnos.

Pero añade una crítica basada en todo el trabajo que tenía que hacer y en la cantidad de correos que recibía:

En cuanto a la labor del profesor, me parece que es uno de los profesores más implicados con su asignatura y que vive por y para ella, se nota muchísimo que le gusta dar clases pero creo que quiere abarcar demasiado y quiere que nosotros abarquemos también demasiado. La verdad es que en más de una ocasión me he agobiado bastante con la asignatura porque hay mucho trabajo que hacer, ojo, me parece genial hacer proyectos, tareas obligatorias, ensayos etc. pero si que es verdad que hay tareas que no he entendido del todo bien y que también esta asignatura ha ocupado gran parte de mi tiempo y me ha quitado tiempo para otras asignaturas asi que al igual que con los grupos al menos reduciría las tareas obligatorias o ponerlas equitativamente por ejemplo, 6 ensayos, 6 tareas obligatorias, el diario y los proyectos, pero no nueve tareas, más los seis ensayos, más los proyectos, el diario y las actividades a las que hemos tenido que ir como el MUAC o la feria de la historia etc. que me ha encantado hacer pero unido a todas las demás tareas ha sido mucho trabajo.

También creo que el profesor debería reducir "muy mucho" los correos que manda, porque no da tiempo a terminar de leer uno cuando ya ha enviado dos más y no podemos abarcar tanto.

\section{Otra alumna española del equipo de ELE para ruso ha- blantes escribe lo siguiente:}

Gracias a este curso he podido relacionarme con personas de otros países y eso me ha enriquecido mucho culturalmente pero definitivamente, lo mejor de este año ha sido poder participar en una metodología tan diferente como la ECO, aprender los conceptos de una manera distinta, haciendo nuestros los conceptos, la asignatura, los proyectos, una experiencia dificil de olvidar. No creo que olvidemos cómo hemos aprendido estos conceptos, no se ha tratado de estudiar todo para vomitarlo después en un examen, ha sido para enriquecernos a nosotros mismos, para crecer como hablantes y, sobre todo, como personas, una manera diferente que nos ha ayudado a estudiar y, sobre todo, nos ha enseñado a cómo estudiar.

Una alumna rusa de ese mismo equipo escribió: 
Aprendí mucho y comencé a comprender más profundamente el progreso lingüístico. Ahora desea aprender más sobre cómo funcionan los idiomas, la gramática y la semántica. Sin duda, después del final del curso, no me detendré en lo que se ha logrado. Leeré más sobre idiomas, veré películas científicas sobre su desarrollo. Sobre todo, me gustaba comparar idiomas, entender la diferencia entre nuestra percepción y, en consecuencia, nuestra cultura. Después de todo, el lenguaje es un espejo, que absorbe y refleja absolutamente todo lo que sucede en la sociedad.

Me gustó que el profesor no nos diera inmediatamente las respuestas a nuestras preguntas. Nos hizo pensar, buscar respuestas por nosotros mismos. No fue fácil. Pero fue muy interesante. Siempre recordaré la diferencia en la dirección del verbo en inglés y en español, precisamente porque tuvimos que resolverlo nosotros mismos.

Voy a terminar con las palabras iniciales del portafolios de una alumna que vino a hablar conmigo uno de los primeros días de clase para decirme que ella iba a optar por el examen ya que no podía trabajar en equipo ni se podía adaptar a la metodología. Me comentó que no iba a tener tiempo porque estaba cursando el Grado en Estudios de Asia Oriental por las mañanas. Por fortuna, pocas semanas después se ofreció a ser moderadora del primer debate sobre las lenguas de España junto al alumno de Derecho. Fue tanto lo que le enriqueció la experiencia que decidió incorporarse a la clase y a la evaluación continua. Estas son las palabras iniciales de su portafolios:

"Recordarás la hora porque en aquel mo-
mento Se acercó a ti quien no se espera nunca”.
Luis Rosales, La casa encendida (1949).

La asignatura de Lingüística de este curso fue, para mí, como eso que dice Luis Rosales que no se espera nunca. Entre todas las clases monótonas, escuchando al profesor, quietos y callados en la silla, durante horas, mirando constantemente al reloj para que ese tiempo estancado pasase, apareció esta asignatura.

Al principio fui un poco escéptica sobre ella, ya que la metodología ECO me resultaba extraña y no estaba convencida de si funcionaría en mí, pero una vez me uní a ella empecé a disfrutar cada vez más, hasta el punto de que me gustaría empezar para llevarla a cabo con mucha más creatividad de lo que lo he hecho. 
A mí también me gustará empezar de nuevo para llevarla a cabo con más creatividad, el curso 2019-2020. El portafolios de esta alumna termina con otra cita de Luis Rosales que expresa muy bien cómo me siento en este momento tras narrar la magnífica experiencia que he vivido y en la que me he enriquecido tanto como el alumnado de Lingüística:

"Porque soy como un niño que despierta en un túnel, Y jamás he sentido la plenitud que estoy sintiendo en este instante". Luis Rosales, La casa encendida (1949).

Jornadas de Formación e Innovación Docente del Profesorado | № 2 (2019) Esta obra se distribuye con la licencia Creative Commons 


\section{Referencias Bibliográficas}

Camacho-Taboada, V. (2019): Design Thinking en asignaturas propedéuticas de primero de Grado. Comunicación presentada a las VI Jornadas de Formación e Innovación Docente. Sevilla, España: Universidad de Sevilla.

Herrero, E. y Mora, J. P. (2018). Aprendizaje Basado en Retos con Design Thinking: ECO en el Grado en Filología Hispánica de la Universidad de Sevilla. Comunicación presentada al I Congreso Iberoamericano de Docentes, Universidad de Cádiz. Algeciras, del 6 al 8 de diciembre 2018.

Mora, J. P. (2014). Creación de un entorno de aprendizaje crítico y significativo en Lingüística, asignatura de primero de filología. Comunicación presentada al I Seminario Iberoamericano de Innovación Docente de la Universidad Pablo de Olavide. Sevilla, 20 y 21 de noviembre 2014.

Mora, J. P. (2015). Sobre el mejor uso del tiempo de clase y las nuevas tecnologías para lograr un aprendizaje más significativo en grupos numerosos de Lingüística, asignatura de primero de Filología. En R. Porlán Ariza y E. Navarro Medina (Coords.), II Jornadas de Docencia Universitaria 2015. Sevilla: Instituto de Ciencias de la Educación / Universidad de Sevilla.

Mora, J. P. (2017). Una experiencia de cambio docente en Filología. En R. Porlán (Coord.), Enseñanza Universitaria: Cómo Mejorarla. Madrid: Editorial Morata.

Torres-Gordillo, J. J., Camacho-Taboada, V. García-Jiménez, J., Herrero-Vázquez, E. A., Mora-Gutiérrez, J. P., y (2019). Aprendizaje como proceso de diseño: innovación universitaria desde el Design Thinking. Comunicación presentada a las I Jornadas de Innovación Docente y Proyectos Estudiantiles Vicerrectorado de Estudiantes. Universidad de Sevilla, del 29 al 31 de mayo 2019. 